\title{
IMPORTANCIA DEL DISEÑO DE LA ESTACIÓN DE TRABAJO Y LA BUENA POSTURA
}

\author{
María del Rosario Párraga V.*
}

\section{RESUMEN}

El factor humano es el componente más importante en todo sistema de trabajo, su seguridad y comodidad son aspectos que deben tenerse presentes para obtener su óptimo desempeño. El diseño de la estación de trabajo debe ser tal que permita lograr una relación entre el usuario y su tarea de forma que éste no se vea perturbado con el equipo que usa, sino por el contrario que lo encuentre útil y facilite su labor además de evitarle posturas incorrectas que le causen lesiones en el desempeño de sus funciones.

Palabras claves: Postura de trabajo, estación de trabajo, fatiga.

\section{ABSTRACT}

The human factor is the most important component in the work system. the security and commodity are aspects that should be remembered to obtain a good performance. The workstation design should be in such a way that let the users and enable them to achieve a relationship so that the users will not be disturbed by the equipment they are using with their work make easier its work. Also, they may avoid inadequate postures that cause injury in operators working functions.

Key words : Position of work, work station, fatigue

\section{INTRODUCCIÓN}

Como ingenieros industriales nuestro interés siempre se ha centrado en el deseo de lograr una mejora y alcanzar elevar la productividad en las tareas que desarrollamos. Hemos centrado nuestra atención en la mejora de métodos de trabajo, la reducción de tiempos, la calidad del trabajo, la minimización de costos, entre otros. Todos estos mecanismos válidos para el logro de nuestros objetivos, pero a su vez, que demandan del trabajador, la flexibilidad para el cambio rápido en la adopción de nuevas formas de trabajo, rapidez y calidad en los productos que elaboran, sus aportes a través de circulos de calidad.

A estos requerimientos por parte del personal hemos respondido con la capacitación para la mejora de sus habilidades, otorgando suplementos, que en suma son necesarios para que el trabajador pueda recuperarse de la fatiga y proporcionarnos un dato real de los tiempos de fabricación, atendiendo las propuestas de cambio que fueran viables. Sin embargo, uno de los aspectos que hemos descuidado es el diseño de la estación de trabajo y la postura

*Ingeniero Industrial. Departamento de Producción y Gestión Facultad de Ingenieria Industrial. UNMSM que se debe adoptar para la ejecución de las tareas, esto quizá porque es obvio en algunos casos, por ejemplo que las herramientas que el trabajador necesita estén a su alcance, o en otros casos, porque es dificil modificar sus costumbres como la de no sentarse derecho o la de cruzar las piernas.

Tales aspectos son de suma importancia, porque contribuyen a elevar la productividad del trabajo aunque este aporte es algo dificil de medir, como por ejemplo cuantificar la comodidad en la estación de trabajo lo que le permitirá estar menos fatigado y por tanto quizá más predispuesto a trabajar, o medir el grado de satisfacción con su estación de trabajo lo que pudiera reflejarse en una mayor identificación con el mismo y así mejore su calidad o rendimiento; o que minimizando el nivel de ruido en su entorno laboral se propiciaría a estar menos estresado y asumir desiciones menos impulsivas y más lógicas.

Lo que si es fácil deducir es que el desarrollo adecuado de las tareas, en términos de adopción de posturas correctas, reducirá el porcentaje de ausencias por dolores de espalda, de cuello, muñecas, pies, etc.; y asimismo se evitará retrasos por estas mismas causas. 


\section{IMPORTANCIA DEL DISEÑO DEL LUGAR DE TRABAJO}

No debemos ignorar que la motivación es un factor determinante en el comportamiento humano y cualquier sistema de trabajo no podría operar exitosamente si el factor humano no persigue el mismo interés y proposito de la organización. Por ésto, para lograr un mejor funcionamiento de un sistema de trabajo se deben implementar medidas que ayuden a la interacción del componente humano y los otros elementos, llámense estos últimos muebles, máquinas, herramientas, iluminación, ruido, etc. Si se presta atención a todos los aspectos del diseño de la estación de trabajo de manera que sean más seguras y agradables al usuario no sólo encontraremos que serán apreciadas por ellos sino que tambien disminuirá la resistencia al esfuerzo que deben desplegar para la ejecución de sus tareas.

Este artículo, se ocupa principalmente de señalar pautas para el diseño y la postura remarcando los aspectos que debemos tener en cuenta para una adecuada interrelación entre el hombre y su estación de trabajo.

\section{DISEÑAR EL LUGAR DE TRABAJO}

Para iniciar el diseño del lugar de trabajo es imprescindible conocer qué tareas se van a desarrollar en ese espacio, decidir si estas pueden hacerse sentado o requieren que el trabajador permanezca de pie. Las dimensiones de los usuarios si es conocida o se debe trabajar con un rango de medidas correspondiente a la población de usuarios. Otro aspecto fundamental para el diseño de la estación de trabajo es la buena postura que debe mantener el usuario en todo momento, de manera que los componentes de la estación de trabajo no deben forzar al operario a adoptar un postura incorrecta sino por el contrario, debe propiciar los cambios posturales adecuados. Uno de los principios básicos es el de mantener la columna derecha, conservando su curvatura natural, los brazos, muñecas y pies en posición neutral, es decir una postura natural sin ángulos forzados como muchas veces encontramos, como por ejemplo al digitar o al operar un pedal. Ver Figuras 1 y 2.

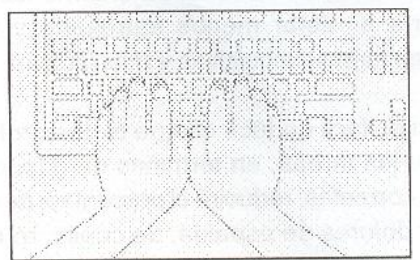

Figura 1. Posición de las muñecas en ángulo forzado

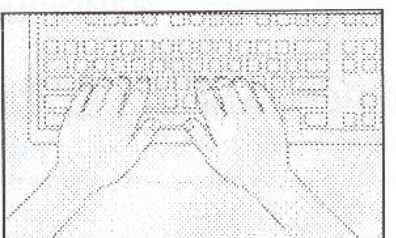

Figura 2. Posición neutral de las muñecas

Los responsables del diseño deben considerar los requerimientos de la labor tanto como las características anatómicas, fisiológicas, antropométricas. La figura 2, presenta un esquema triangular de la postura de trabajo con relación a 3 variables: las características del usuario, tales como sus dimensiones corporales, su forma, sus capacidades físicas, etc. los requerimientos de la tarea como conocimientos técnicos y el diseño del espacio de trabajo.

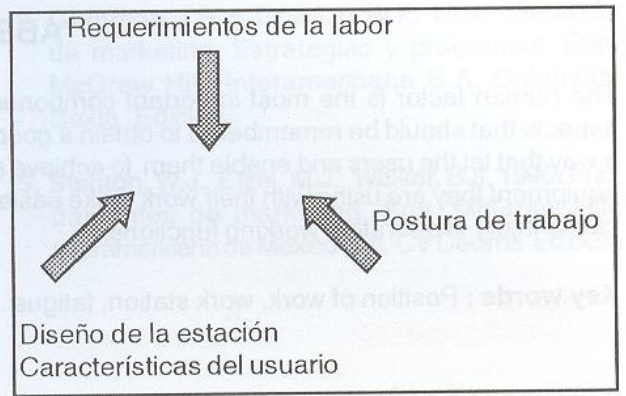

Figura 3. Esquema triangular de la postura de trabajo.

Las dimensiones de los muebles a ser utilizados por lo general deben ser diseñados de manera que satisfagan cómodamente al $90 \%$ de la población de usuarios.

Los problemas surgen cuando los usuarios pertenecen a los extremos de la población, como por ejemplo, aquel individuo extremadamente alto, o bajo u obeso; en estos casos es necesario hacer arreglos especiales para poder acomodarlos. Por lo general se busca que los muebles a utilizar sean facilmente acondicionables a las diferentes longitudes corporales de los usuarios; la silla debe ser regulable tanto en lo que se refiere a la altura del asiento como a la posición del respaldo que debe poder ajustarse para dar soporte a la región lumbar.

Si se trabaja de pie, una superficie de trabajo muy baja provocará una mala postura, pues el usuario tenderá a inclinarse para poder encontrar un punto de apoyo en sus antebrazos o codos. Una superficie de trabajo alta también provocará que el trabajador realice un esfuerzo innecesario al mantener sus brazos en alto para realizar su labor, lo que requiere 
Cuadro 1. Factores que influyen en la postura de trabajo

\begin{tabular}{|c|c|c|}
\hline a. & $\begin{array}{ll}\text { Características del usuario } & 1 \\
& 2 \\
& 3 \\
& 4 \\
5 \\
6 \\
7 \\
8 \\
9 \\
1\end{array}$ & $\begin{array}{ll}1 & \text { Edad } \\
2 & \text { Dimensiones de sus segmentos corporales } \\
3 & \text { Peso } \\
4 & \text { Condición física } \\
5 & \text { Rango de movilidad de sus segmentos corporales } \\
6 & \text { Problemas músculo esqueléticos } \\
7 & \text { Lesiones } \\
8 & \text { Grado de visión } \\
9 & \text { Habilidades manuales } \\
10 & \text { Obesidad }\end{array}$ \\
\hline b. & Requerimientos de la labor & $\begin{array}{l}11 \text { Requerimientos visuales } \\
12 \text { Esfuerzos que demanda la labor } \\
13 \text { Tiempo de Ciclo del trabajo } \\
14 \text { Periodos de descanso } \\
15 \text { Conocimientos técnicos } \\
16 \text { Riesgos probables } \\
17 \text { Exigencias de precisión y calidad } \\
18 \text { Grado de concentración exigible }\end{array}$ \\
\hline c. & Diseño de la estación de trabajo 1 & $\begin{array}{l}19 \text { Dimensiones de la silla } \\
20 \text { Dimensiones de la superficie } \\
21 \text { Diseño de la silla } \\
22 \text { Niveles de iluminación } \\
23 \text { Apoyo para los pies }\end{array}$ \\
\hline
\end{tabular}

un gasto energético mayor. La altura adecuada para la superficie de trabajo es de $5 \mathrm{~cm}$ debajo del codo, por cuanto es necesario tener en cuenta que para establecer esta altura se debe elegir el rango superior de la población de usuarios y en el caso de personas de baja estatura se les puede proporcionar plataformas de manera que alcancen el nivel óptimo señalado.

\section{FACTORES QUE INFLUYEN EN LA POSTURA DE TRABAJO}

En el cuadro 1 se señalan algunos factores que influyen en la póstura de trabajo.

\section{CONCLUSIONES}

Debemos considerar los beneficios que trae consigo el diseño de la estación de trabajo y la adopción de posturas adecuadas tanto a los trabajadores como a la dirección. A los primeros, les traerá co- modidad, facilitará el desarrollo de sus tareas y los protegerá de posibles lesiones fisicas. Los segundos, se verán beneficiados, por la reducción en la fatiga de su personal, la motivación generada por un lugar de trabajo confortable, la reducción en los gastos ocasionados por lesiones en el puesto de trabajo, como el ausentismo que provoca retrasos. Cabe señalar que muchos de los cambios que deben hacerse para lograr un buen diseño de la estación de trabajo no implican grandes inversiones sino buena voluntad.

\section{BIBLIOGRAFÍA}

1. Bridger, RS. 1995. Introduction to Ergonomics. Edit. Mc Graw Hill. New York.

2. Konz, S. 1992. Diseño de Sistemas de Trabajo. Edit. Limusa. México.

3. Oborne, D.J. 1999. Ergonomía en Acción. Edit. Trillas. México. 